\title{
ACTIN7 Is Required for Perinuclear Clustering of Chloroplasts during Arabidopsis Protoplast Culture
}

\author{
Michael B. Sheahan, David A. Collings ${ }^{\circledR}$, Ray J. Rose and David W. McCurdy *(1) \\ School of Environmental and Life Sciences, The University of Newcastle, Callaghan, NSW 2308, Australia; \\ Michael.Sheahan@newcastle.edu.au (M.B.S.); David.Collings@newcastle.edu.au (D.A.C.); \\ Ray.Rose@newcastle.edu.au (R.J.R.) \\ * Correspondence: David.McCurdy@newcastle.edu.au; Tel.: +61-(2)-4921-5879
}

Received: 5 January 2020; Accepted: 7 February 2020; Published: 10 February 2020

\begin{abstract}
In Arabidopsis, the actin gene family comprises eight expressed and two non-expressed $A C T I N$ ( $A C T$ ) genes. Of the eight expressed isoforms, ACT2, ACT7, and ACT8 are differentially expressed in vegetative tissues and may perform specific roles in development. Using tobacco mesophyll protoplasts, we previously demonstrated that actin-dependent clustering of chloroplasts around the nucleus prior to cell division ensures unbiased chloroplast inheritance. Here, we report that actin-dependent chloroplast clustering in Arabidopsis mesophyll protoplasts is defective in act7 mutants, but not act2-1 or act8-2. ACT7 expression was upregulated during protoplast culture whereas $A C T 2$ and $A C T 8$ expression did not substantially change. In act2-1, ACT7 expression increased in response to loss of $A C T 2$, whereas in act7-1, neither $A C T 2$ nor ACT8 expression changed appreciably in response to the absence of ACT7. Semi-quantitative immunoblotting revealed increased actin concentrations during culture, although total actin in act7-1 was only two-thirds that of wild-type or act2-1 after $96 \mathrm{~h}$ culture. Over-expression of ACT2 and ACT8 under control of ACT7 regulatory sequences restored normal levels of chloroplast clustering. These results are consistent with a requirement for ACT7 in actin-dependent chloroplast clustering due to reduced levels of actin protein and gene induction in act7 mutants, rather than strong functional specialization of the ACT7 isoform.
\end{abstract}

Keywords: actin; ACTIN genes; Arabidopsis; chloroplast clustering; protoplast culture

\section{Introduction}

The actin cytoskeleton facilitates numerous cellular processes required for the correct functioning and development of multicellular eukaryotes. Unlike yeast, where actin is encoded by a single gene [1], in multicellular eukaryotes, actins are encoded by multi-gene families. In Arabidopsis thaliana (Arabidopsis), eight expressed actin isoforms exist [2]. Based on their phylogenetic relationship and expression pattern, Arabidopsis actins are classified as either vegetative or reproductive, with each class being expressed predominantly in vegetative or reproductive tissues, respectively. The vegetative class of actins comprises ACT2, ACT7, and ACT8. Interestingly, the sequence divergence between individual actin isoforms in plants is greater than the divergence between non-muscle and muscle isoforms of actin in animals, suggesting the potential for plant actin isoforms to perform discrete functions within the cell $[3,4]$. Alternately, such divergence may simply reflect developmental rather than intracellular specialization. While the number of actin genes varies dramatically between different plant species, with the eudicot Medicago truncatula containing only four actin genes but with the monocot Zea mays containing 21 separate genes, the division of actins into vegetative and reproductive classes is broadly conserved across plant species $[4,5]$. 
In animal and fungal cells, different actin isoforms can perform different functions, not only between the major classes of muscle and non-muscle actin but even within these classes, with these conclusions derived from a range of molecular and biochemical experiments [6], green fluorescent protein (GFP)-fusion studies [7] and mutant analyses [8]. Evidence for isoform-specific functions of plant actins is strongest for the vegetative actin ACT7. During tissue culture, the ACT7 gene is strongly induced by auxin and is required for callus formation, whereas formation of callus proceeds normally in an ACT2 knockout mutant [9]. ACT7 mutants also show delayed germination and altered root growth, possibly explaining the strong selective disadvantage of act7-4 when grown in competition with wild-type plants [10]. Nuclear migration and positioning to establish polar outgrowth of root hairs requires ACT7 [11], whereas ACT2 is required for bulge site selection and tip growth [12]. Furthermore, an ACT2-dependent defect in root hair growth could not be complemented by overexpression of ACT7 in the act2 mutant [13]. Bacterially-expressed ACT2 and ACT7 display distinct biochemical properties such as kinetics of filament polymerization and interaction with actin-binding proteins such as profilin [14]. Recently, experiments using novel GFP-actin fusions demonstrated that ACT2 and ACT7 generate distinct and cell-type-specific filamentous arrays, either forming isoform-specific filaments or various mixed-polymer filaments depending on the cell type [15]. Collectively, these studies point towards functional specificity of the actin cytoskeleton in plants by expressing functionally different actin isoforms [3].

Progress in investigating potential isoform-specific functions of plant actins has been limited by the paucity of point mutations in different $A C T$ genes. Unlike the situation with tubulin and microtubules, where extensive collections of tubulin mutants with either recessive or semi-dominant growth phenotypes exist in both Arabidopsis [16,17] and other species including rice [18] and tef [19], few actin mutants have been described. However, in recent years several actin mutants have been characterized in Arabidopsis and other species. These include the dominant negative act2-2D mutant in ACT2 which showed disruption not just in root hair growth but also in diffusely elongating cells, the recessive der 1 mutants in ACT2 in which different point mutations all result in defects in root hair growth [12] and changes in overall growth of the plant [20], and the dominant-negative fiz1 point mutation in ACT8 which results in fragmentation of the actin cytoskeleton and disrupted organelle trafficking [21].

During culture of tobacco mesophyll protoplasts, chloroplasts move from the cortical cytoplasm and reposition to the perinuclear region [22]. This process of chloroplast clustering to the nucleus ensures that when the protoplasts subsequently divide, unbiased inheritance of the chloroplast population to each daughter cell is achieved [22,23]. Experiments with inhibitors of either microtubule or actin filament (AF) polymerization indicated that this repositioning is an AF- and not microtubule-dependent phenomenon [22]. Time-lapse imaging suggested that chloroplast movements from the peripheral cytoplasm to the perinuclear region may involve the capture of individual chloroplasts in an actin network, which itself undergoes dynamic repositioning [23]. This process of bulk repositioning of chloroplasts enmeshed in a dynamic network of actin is distinct from the more conventional models whereby dynamic chloroplasts move along stationary AFs or bundles [24]. Once repositioned to the perinuclear region, however, the mechanism that results in the chloroplasts being maintained there remains unknown. A likely possibility is that the chloroplasts are trapped somehow by specific interaction with the "nuclear basket" of AFs, which is a common component of the actin cytoskeleton in plant cells $[3,22,24]$.

In this study, we have investigated the process of chloroplast clustering in the model species Arabidopsis. We show that as with tobacco, chloroplasts in cultured Arabidopsis mesophyll protoplasts undergo chloroplast clustering to the perinuclear region prior to cell division, and that this process is dependent on the actin cytoskeleton but not microtubules. Chloroplast clustering was completely absent in two mutant alleles of $A C T 7$, act7-1, and act7-4, but was essentially unaffected in mutants of the other vegetative actins. While reverse transcription-quantitative polymerase chain reaction (RT-qPCR) and immunoblotting demonstrated that both $A C T 7$ expression and total actin accumulated during 
protoplast culture, this did not happen in the act7 mutants and total actin levels remained low. Further, as over-expression of $A C T 2$ and ACT8 under control of the ACT7 promoter restored full chloroplast clustering, this indicates that the loss of chloroplast clustering in the act7 mutants is probably due to reduced levels of total actin. These results are discussed in terms of the mechanism of actin-dependent chloroplast clustering and the failure of this process in the act7 mutants.

\section{Results}

\subsection{Actin-Dependent Clustering of Chloroplasts during Protoplast Culture}

Prior to the first cell division in cultured tobacco mesophyll protoplasts, chloroplasts move via an actin-dependent process from the cortical cytoplasm and cluster around the surface of the centrally-placed nucleus [22]. We confirmed that a similar relocation and perinuclear clustering of chloroplasts occurred in cultured Arabidopsis mesophyll protoplasts (Figure 1). Immediately following protoplasting, chloroplasts were present primarily in the cortex of protoplasts (Figure 1a) but over the course of four days they relocated through intermediate localizations (Figure 1b) to a primarily perinuclear location (Figure 1c). These stages of re-localization were quantified in wild-type protoplasts (Figure 1d) and this pattern was not affected by the expression of the fluorescent F-actin marker GFP-fABD2 (a fusion of GFP with actin binding domain 2 of Arabidopsis AtFIM1 [25]) (Figure 1f).
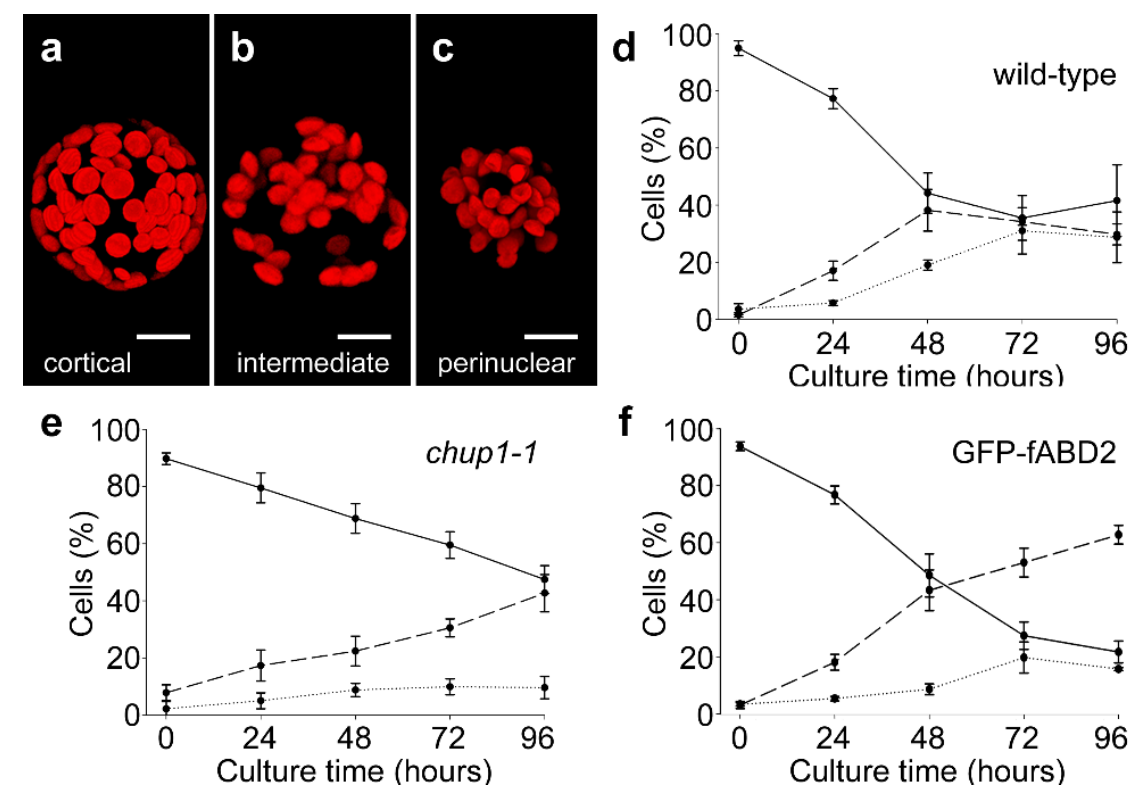

Figure 1. Analysis of chloroplast clustering in Arabidopsis protoplasts. (a-c) Images of chloroplasts (red autofluorescence) in either the (a) cortical, (b) intermediate, or (c) perinuclear distributions in cultured protoplasts. (d-f) Quantitative analysis of the repositioning of chloroplasts from cortical to perinuclear across $96 \mathrm{~h}$ of culture. (d) Wild-type protoplasts. (e) chup1-1 protoplasts. (f) Protoplasts from a GFP-fABD2 line. For $\mathrm{d}-\mathrm{f}$, solid lines, dashed lines and dotted lines represent cortical, intermediate and perinuclear distributions of chloroplasts, respectively. Scale bars in a-c $=10 \mu \mathrm{m}$. Data in d-f is mean $\pm \mathrm{SE}, n=3-5$ experiments.

To examine whether the mechanism of chloroplast clustering in cultured protoplasts is similar to light-dependent chloroplast repositioning, we analyzed clustering in mesophyll protoplasts isolated from homozygous mutants of CHUP1. CHUP1 is required for light-dependent repositioning of chloroplasts from anticlinal to periclinal cell walls of leaf mesophyll cells exposed to different intensities of blue light [26]. In the chup1-1 mutant, perinuclear clustering of chloroplasts continued to occur in cultured mesophyll protoplasts (Figure 1e), with this being only slightly slower than wild-type 
(Figure 1d). This result indicates that the mechanisms for repositioning chloroplasts in mesophyll cells in response to the stimuli of high light and protoplast culture are substantially different.

As found in tobacco, culturing Arabidopsis protoplasts in the presence of the actin disrupter latrunculin $\mathrm{B}(1 \mu \mathrm{M})$ prevented relocation of chloroplasts from the cortex, demonstrating that relocation is dependent on the actin cytoskeleton. In contrast, microtubule de-polymerization with $10 \mu \mathrm{M}$ oryzalin slowed but did not block the clustering response (Figure 2a). To test the involvement of different actin isoforms in mediating chloroplast clustering, we performed similar analyses using T-DNA insertional mutants of all three Arabidopsis vegetative actins (ACT2, ACT7, and ACT8) and one reproductive actin (ACT11). ACT2 and ACT7 are the most divergent vegetative actins, while ACT8 is closely similar to ACT2. Each insertion line shows substantial or complete reduction in the levels of the respective $A C T$ transcript and protein product in seedling tissue $[9,10,13,27]$. When mesophyll protoplasts from each line were cultured for up to five days, chloroplast clustering in act2-1 and act11-1 protoplasts occurred to the same extent as in wild-type protoplasts (Figure 2b), whereas no clustering was evident in protoplasts from the ACT7 alleles, act7-1 and act7-4 (Figure 2b). Clustering did occur in act8-2 protoplasts, but at a slower rate than the wild-type. Overall, these results suggest that the phenomenon of chloroplast clustering relies on the presence of functional ACT7 protein.
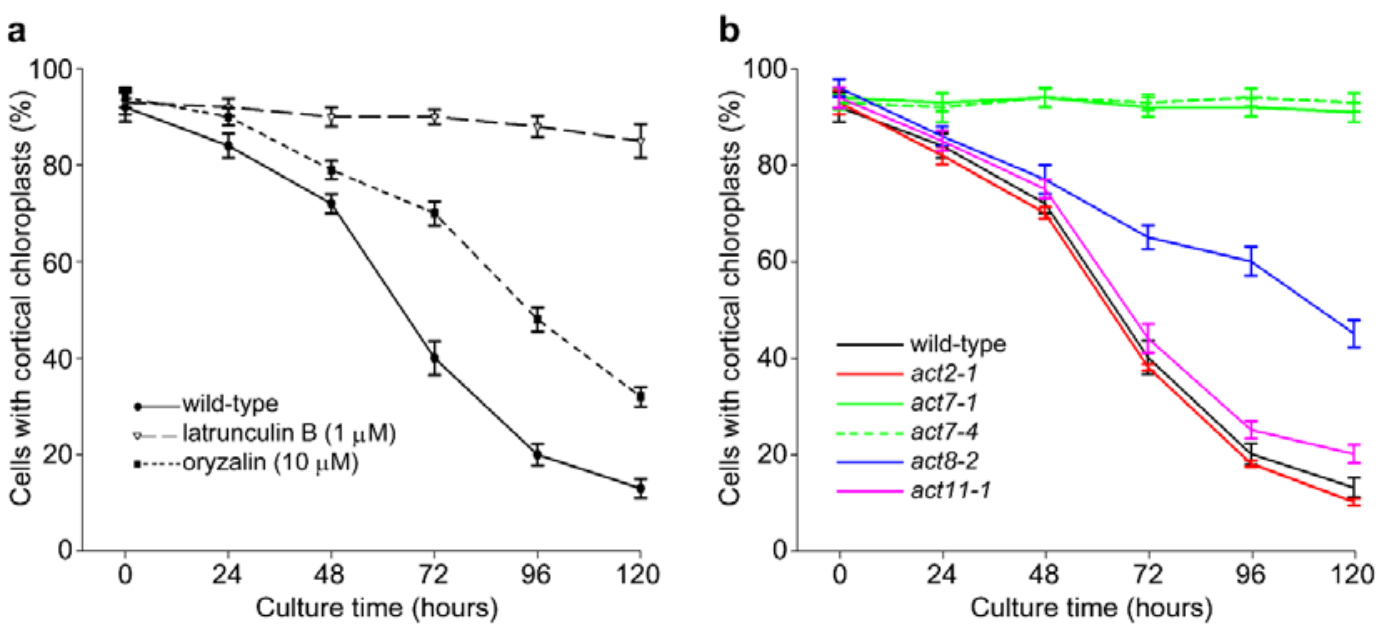

Figure 2. Chloroplast clustering in Arabidopsis protoplasts is actin-dependent and disrupted in act7 mutants. (a) Clustering of chloroplasts occurs in Arabidopsis protoplasts. Latrunculin B $(1 \mu \mathrm{M})$ inhibited clustering, showing actin-dependence, whereas oryzalin $(10 \mu \mathrm{M})$ showed that clustering is independent of microtubules. (b) Clustering of chloroplasts around the nucleus is unaffected in act2-1 or act11-1 protoplasts, is completely inhibited in act7 alleles, act7-1 and act7-4, and is slowed in act-8-2 protoplasts. Data is mean $\pm \mathrm{SE}, n=3-5$ experiments.

To determine whether the architecture of the actin cytoskeleton was compromised in the actin mutants, we crossed act2-1, act7-4, and act8-2 plants with a line expressing GFP-fABD2 [25]. Analysis of protoplasts cultured for $48 \mathrm{~h}$ showed that actin organization was largely intact in act2-1 and act7-4 compared to wild-type, but that act8-2 revealed larger bundles of cortical actin. In all lines except act8-2, a finer cortical actin network was apparent along with larger subcortical bundles of AFs (Figure 3). 

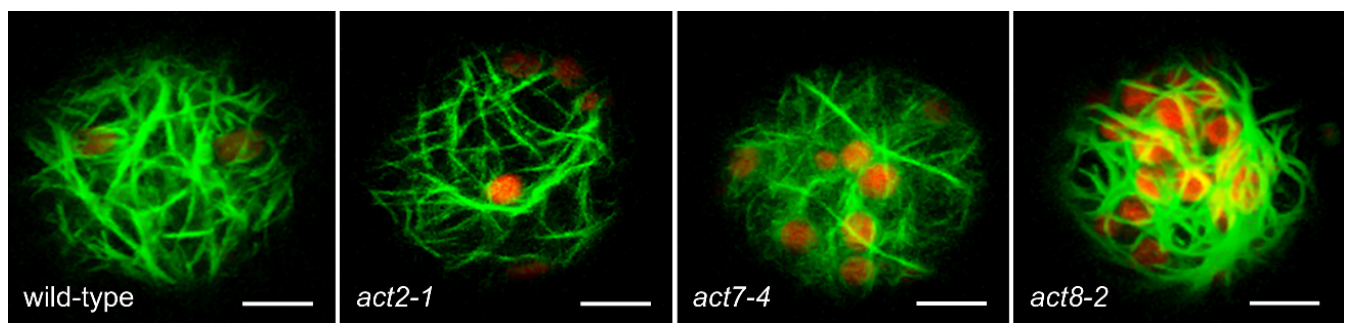

Figure 3. Actin networks in wild-type and act mutants. Images are maximum projections of optical sections ( $4 \mu \mathrm{m}$ deep) of 48 -h protoplasts derived from wild-type (Col-0) and act mutants expressing GFP-fABD2. Red is chlorophyll autofluorescence from chloroplasts. Scale bars $=10 \mu \mathrm{m}$.

\subsection{ACT7 Is Up-Regulated before Re-Initiation of Cell Division}

To assess temporal changes in $A C T$ gene expression across protoplast culture, real-time qPCR was performed (Figure 4). Of the three vegetative actin genes, ACT7 expression in wild-type (Col-0) was substantially increased (over six-fold) over $96 \mathrm{~h}$ of protoplast culture, whereas ACT2 and ACT8 levels did not change appreciably. In act7-1 mutant protoplasts, ACT2 and ACT8 expression did not change in response to the loss of $A C T 7$, whereas in the act2-1 mutant, expression of $A C T 7$ and to a lesser extent ACT8 increased to some degree in response to the lack of ACT2 (Figure 4). These results show that $A C T 7$ transcription increases as cells prepare to re-enter the cell cycle and suggests it is change in $A C T 7$ expression that contributes to the perinuclear clustering of chloroplasts in cultured protoplasts.
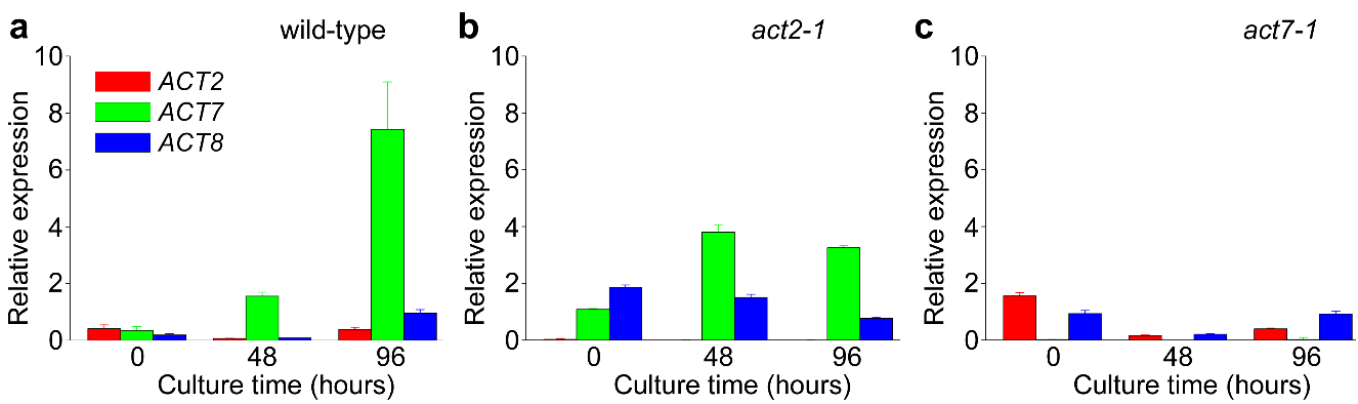

Figure 4. Changes in relative expression of $A C T 2, A C T 7$, and $A C T 8$ across culture of protoplasts derived from (a) wild-type and (b) act2-1 and (c) act7-1 mutants. Data is mean $\pm \mathrm{SE}, n=3$ experiments.

\subsection{Analysis of Total Actin}

Given the changes in gene expression documented in Figure 4, semi-quantitative immunoblotting was performed to compare total levels of actin in wild-type compared to act mutants. As isoform-specific anti-actins that distinguish between ACT7, ACT2, and ACT8 polypeptides are not available, see [24], total actin in protoplasts was assessed using the $\mathrm{C} 4$ monoclonal anti-chicken gizzard actin antibody [28]. In freshly isolated protoplasts, total actin levels in the act2-1 and act7-1 mutants were substantially decreased compared to wild-type (Figure 5). Across subsequent protoplast culture, total actin levels in wild-type protoplasts increased in a linear fashion, whereas total actin levels in the act2-1 mutant was about two-thirds that of wild-type at $48 \mathrm{~h}$ but equaled wild-type levels by $96 \mathrm{~h}$ culture. In contrast, increase in total actin levels in act7-1 plateaued by $48 \mathrm{~h}$ and remained at about two-thirds that of both wild-type and act $2-1$ by $96 \mathrm{~h}$ (Figure 5). This result shows that total actin levels in the act7-1 mutant did not respond to protoplast culture in the same way as wild-type and act2-1, suggesting that the chloroplast clustering phenotype seen in the act 7 mutants is most likely due to decreased total protein levels in the mutant rather than resulting from an isoform-specific function of ACT7. Interestingly, while total actin levels in both act2-1 and act7-1 were similarly decreased compared to wild-type at $48 \mathrm{~h}$, the reduction in total actin levels in act2-1 had no effect on chloroplast clustering at $48 \mathrm{~h}$ whereas a similar reduction in total act7-1 was associated with an inhibition of chloroplast clustering (Figure 2). 


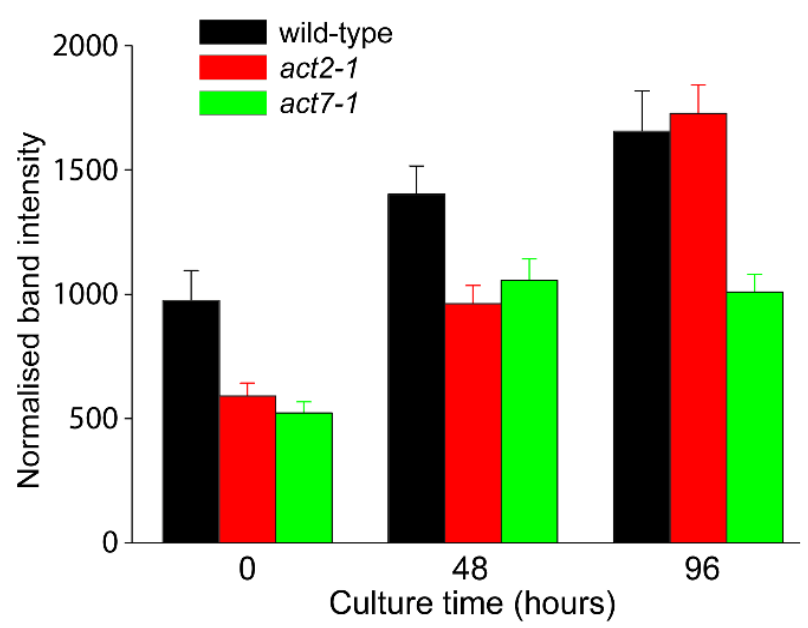

Figure 5. Semi-quantitative immunoblotting of total actin levels across culture of protoplasts derived from wild-type (Col-0), act2-1, and act7-1. Total protein was extracted from cultured protoplasts at the indicated time points and immunoblotting was performed with $\mathrm{C} 4$ anti-actin monoclonal antibody. Normalised band intensities were determined by duplicate immunoblots probed with anti-ß-tubulin. Data is mean $\pm \mathrm{SE}, n=3$ experiments.

\subsection{Over-Expression of Vegetative Actins Suppresses the Act7-4 Phenotype}

Given the suggestion from gene expression and total protein data that reduced actin levels within the act7 mutants may cause the chloroplast clustering phenotype, we tested whether over-expression of the two vegetative actins $A C T 2$ and $A C T 8$, under the control of the ACT7 promoter and terminator sequences [24], can complement the act7 phenotype. Analysis of different lines in which ACT7p::ACT2 and $A C T 7 p:: A C T 8$ constructs were expressed in the act $7-4$ mutant showed that over-expression of either ACT2 or ACT8 restored normal chloroplast clustering in the act7-4 mutant compared to wild-type (Figure 6). This result is consistent with the interpretation that it is the actin protein concentration itself, rather than a functional dependence-specific actin isoform, that causes the disrupted chloroplast clustering phenotype in the act7-1 and act7-4 mutants.

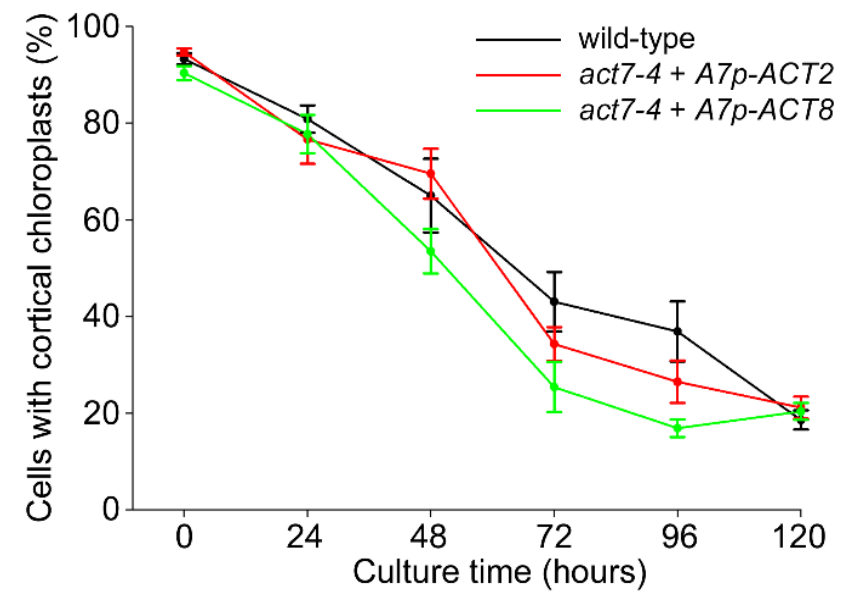

Figure 6. Over-expression of the vegetative actins $A C T 2$ and ACT8 suppresses the act7-4 phenotype. Chloroplast clustering is indistinguishable between wild-type or two transgenic lines whereby ACT2 and ACT8 are expressed in the act7-4 mutant under control of the ACT7 promoter. Data is mean $\pm \mathrm{SE}$, $n=3$ experiments. 


\section{Discussion}

The perinuclear clustering of chloroplasts which occurs prior to cell division in protoplasts provides a mechanism to ensure unbiased inheritance of the chloroplast population to both daughter cells. This process was first reported in tobacco mesophyll protoplasts [22] and this report now establishes that the same phenomenon occurs in mesophyll protoplasts from Arabidopsis leaves. Furthermore, as found in tobacco, the actin inhibitor latrunculin B strongly prevented chloroplast clustering from occurring, indicating that the process in Arabidopsis protoplasts is also actin-dependent.

The mechanism of actin-dependent chloroplast repositioning during protoplast culture is not known. Time-lapse movies of tobacco protoplasts expressing GFP-fABD2-labelled AFs showed chloroplasts enmeshed within an actin network, with localized movement of the actin network itself appearing to be the driving force for re-location of the chloroplasts, rather than movement of chloroplasts along stationary actin bundles. Furthermore, chloroplasts within the perinuclear region appeared to be enmeshed within a static perinuclear actin network [23]. These observations are distinct from descriptions of other examples of chloroplast movement in which chloroplasts enmeshed in actin baskets nonetheless translocate along stationary AFs [24]. Light-dependent re-location of actin bundles is reported to involve short, chloroplast-associated filaments, described as chloroplast actin, which assemble at the leading edge of chloroplasts [29] and interact with the plasma membrane to relocate chloroplasts in response to light, reviewed in [30]. Interestingly, the presence of this chloroplast actin and its interaction with the plasma membrane is dependent on CHUP1, a multifunctional protein required for proper chloroplast positioning and photo-relocation movements [26]. Since the process of chloroplast clustering observed here was not substantially disrupted in the chup1 mutant, the mechanism involved in chloroplast clustering must be different from light-dependent chloroplast relocation.

We tested the involvement of individual actin isoforms in the process of chloroplast clustering in Arabidopsis by analyzing various $A C T$ knockout mutants. Being insertional mutants into the promoters, these mutants have a considerable or complete reduction in the levels of their respective $A C T$ transcripts and protein products $[9,10,13,27]$. Somewhat surprisingly, clustering was entirely absent in two alleles of act7 (act7-1 and act7-4) but was unperturbed in act2-1 and act11-1 and only slowed in act8-2. The organization of the actin network was not substantially different to that of wild-type in transgenic lines expressing the GFP-fABD2 marker for actin filaments (Figure 3).

$A C T 7$ is a functionally diverse vegetative actin. Unlike $A C T 2$, which is constitutively expressed in the vegetative tissues of adult plants, $A C T 7$ is expressed more strongly in younger tissues, and is responsive to external stimuli and hormones $[3,4,9,13]$. This ability for ACT7 to show highly regulated expression is consistent with the strong upregulation of its expression during protoplast culture. A consequence of this variable expression is also that the various act7 mutants are defective in aspects of root growth and development $[10,11]$. Our quantitative gene expression analysis showed that $A C T 7$ was the dominant $A C T$ gene expressed across culture of wild-type protoplasts (Figure 4) and that the expression of $A C T 7$ along with $A C T 8$ partially compensated for the absence of $A C T 2$ in the act2-1 mutant. In contrast, neither $A C T 2$ nor $A C T 8$ expression changed substantially from wild-type to compensate for the loss of $A C T 7$ (Figure 4). This observation suggests that the absence of chloroplast clustering in the act7 mutants was due to the absence of any compensatory expression of ACT isoforms in act7. This conclusion is supported by the semi-quantitative immunoblotting (Figure 5), which demonstrated that total actin abundance increased across protoplast culture in wild-type but that actin levels in act7-1 were about two thirds that of wild-type. Thus, the absence of compensatory ACT expression resulting in reduced total actin levels in act7-1 may be the explanation for loss of chloroplast clustering in this mutant.

Our conclusion that it is a reduction in overall actin concentration, rather than the functional specialization of actin isoforms, that is responsible for the inhibition of chloroplast clustering in act7 mutants is also supported by the over-expression experiments in which the two vegetative actins, ACT2 and ACT8, were expressed in the act7-4 mutant under control of ACT7 regulatory sequences. 
In both cases, chloroplast clustering recovered to wild-type levels across $120 \mathrm{~h}$ of protoplast culture (Figure 6). This result, along with the total actin levels reported by immunoblotting, clearly establishes that the absence of chloroplast clustering in act7 mutants is due to reduced levels of total actin in act7 protoplasts rather than being caused by isoform-specific, functional specialization of ACT7. A similar conclusion was reached following experiments in which the sensitivity of root elongation and root swelling to low doses of latrunculin was tested in wild-type and act mutant lines. Wild-type plants showed reductions in root elongation and root tip swelling following $48 \mathrm{~h}$ treatments with latrunculin B concentrations of $100 \mathrm{nM}$ and higher (data not shown), equivalent to a previous report [31]. In act7-1 and act2-1, however, induced swelling and reduced elongation responses were observed at concentrations as low as $30 \mathrm{nM}$. Importantly, preliminary experiments have shown that in ACT7p::ACT2 plants in which $A C T 2$ was expressed under control of the $A C T 7$ promoter, the mutant phenotype was complemented with plants showing wild-type responses to latrunculin treatments (data not shown). Thus, the disrupted chloroplast clustering phenotype in the act7 mutants is not the only phenotype that can be rescued, suggesting a common mechanism based on the total levels of ACT protein.

This ability to complement act mutant phenotypes by over-expression of other ACT genes, suggesting functional interchangeability of ACT proteins, has previously been demonstrated in Arabidopsis. For example, the act7-4 act8-2 double mutant shows a strong and diverse growth phenotype: over-expression, however, of protist and animal cytoplasmic ACT genes under control of the ACT2 promoter complemented the mutant phenotype showing that these divergent actins retained functional competence, although animal muscle actin did not have this ability [3]. These results all suggest that the vegetative actin genes, $A C T 2, A C T 7$, and $A C T 8$, which are expressed in Arabidopsis, need not have any functional specializations. Instead, their role might be to show different patterns of expression within the plant and differences in responsiveness to external stimuli. A counter view to this conclusion, however, is that functional differences do exist between vegetative actin isoforms. This has been suggested based on observations of biochemical differences between ACT2 and ACT7 [14], and the structurally different arrays and filaments formed by these two vegetative actins [15]. Moreover, while our data broadly support the concept that it is total actin levels rather than isoform functional specialization that are responsible for the act 7 mutant phenotype, the lack of a phenotype in the act2-1 mutant even when total ACT levels are low may suggest some subtle functional differences between the different isoforms.

The observation that total actin levels are responsible for the act7 clustering phenotype presumably also has implications for the mechanism of chloroplast clustering. As stated previously, the collective movement of chloroplasts enmeshed within a dynamic actin network [23] is different from other models of actin-dependent motility in plant cells [24,30]. In this regard, the reduction in total actin levels seen in the act7-4 mutant may be a critical feature that does not support differential enmeshment of chloroplasts undergoing clustering, and the more static network of perinuclear actin which appears to be responsible for trapping motile chloroplasts at the nuclear surface. Presumably, diverse activities of actin-binding proteins interacting with sub-domains of the actin cytoskeleton in cultured protoplasts may be the mechanistic element responsible for this process, however minimal levels of total actin in each protoplast is required to maintain differential organization of the actin cytoskeleton and thus execute the ability to differentially orchestrate organelle movements as seen in the example of chloroplast clustering.

\section{Materials and Methods}

\subsection{Plant Growth, Protoplast Isolation and Culture and Reagents}

Arabidopsis thaliana (Arabidopsis) seeds (Col-0) were surface sterilized in $70 \%(v / v)$ ethanol for $1 \mathrm{~min}$ followed by a 1:4 dilution of White King ${ }^{\mathrm{TM}}$ commercial bleach for $5 \mathrm{~min}$, then extensive washed in sterile water and suspended in $0.1 \%(w / v)$ agar solution. The seeds were then distributed evenly over the surface of Petri dishes containing half-strength Murashige and Skoog medium supplemented 
with $1 \%(w / v)$ sucrose and solidified with $1.2 \%(w / v)$ Bacto agar. Plates were placed at $4{ }^{\circ} \mathrm{C}$ for 2 days before being placed horizontally in a controlled growth environment of $16 / 8 \mathrm{~h}$ day/night $\left(22^{\circ} \mathrm{C} / 18^{\circ} \mathrm{C}\right)$ and fluence rate of $90-120 \mu \mathrm{mol} \mathrm{m}{ }^{2} \mathrm{~s}^{1}$. After 10-14 day growth, the plates were placed in complete darkness for $24 \mathrm{~h}$ to reduce starch levels in chloroplasts. The aerial portion of plants was then excised with sterile scissors, placed on sterile paper towel, and coarsely macerated with a sterile razor blade. Protoplasts were then isolated from the macerated leaf tissue and cultured for up to $120 \mathrm{~h}$ as described previously for tobacco mesophyll protoplasts [22]. All reagents were purchased from Sigma-Aldrich (Sydney, NSW, Australia) unless specified otherwise. Oryzalin (Crescent Chemical, Singapore) and latrunculin were prepared as $1000 \times$ stocks in dimethylsulfoxide (DMSO), and diluted to $10 \mu \mathrm{M}$ and $1 \mu \mathrm{M}$, respectively. Protoplast cultures were exposed throughout the culture period to the various drugs, using $0.1 \%(v / v)$ DMSO as control.

Different act insertional mutant lines (obtained from Prof. Richard Meagher, University of Georgia) were crossed with a line expressing GFP:fABD2 [25]. The chup1-1 mutant was generously supplied by Prof. Masamitsu Wada (Kyushu University).

\subsection{Microscopy}

Protoplasts were placed in welled slides in a 2:1 ratio with $0.5 \%(w / v)$ agarose (agarose type VII, Sigma) before applying a coverslip. Images of protoplasts were acquired as z-series with a $1 \mu \mathrm{m}$ interval using a Zeiss LSM510 confocal laser-scanning microscope equipped with a $40 \times$ water-immersion objective. GFP was viewed with $488 \mathrm{~nm}$ excitation and barrier filter selecting 500 to $530 \mathrm{~nm}$, while chloroplast autofluorescence was viewed with 543-nm excitation and a long-pass filter selecting above $650 \mathrm{~nm}$.

\subsection{Reverse Transcription-Quantitative PCR (RT-qPCR)}

Protoplasts of the various lines under investigation were cultured for the indicated times, then $8 \times 10^{4}$ protoplasts were pelleted and dissolved in RLT buffer for total RNA isolation using the Qiagen Plant RNeasy kit (Qiagen, Hilden, Germany). Synthesis of cDNA from $1 \mu \mathrm{g}$ of total RNA was performed using a Superscript III kit (Invitrogen, Waltham, MA, USA) following the manufacturer's instructions. Gene expression was analyzed by RT-qPCR using a RotorGene-Q (Qiagen). PCR reactions were carried out using UBC21 (At5G25760) as the reference gene. PCR master mixes were prepared with Platinum Taq (Invitrogen) using the provided buffer supplemented with $1.5 \mu$ M SYTO9 (Invitrogen), $3 \mathrm{mM}$ dNTPs and $0.4 \mu \mathrm{M}$ of each primer. The qRT-PCR cycling conditions comprised an initial denaturation at $95{ }^{\circ} \mathrm{C}$ for $2 \mathrm{~min}$ followed by 40 cycles of $95^{\circ} \mathrm{C}$ for $10 \mathrm{~s}, 60^{\circ} \mathrm{C}$ for $30 \mathrm{~s}$ and $72{ }^{\circ} \mathrm{C}$ for $30 \mathrm{~s}$. For each gene analyzed, two biological and three technical repetitions were performed. Data analyses were performed using Q-Gene software [32-34]. Q-Gene software uses mean normalized data and the $\Delta \Delta C_{\mathrm{T}}$ method to calculate relative expression (the calibrator was the lowest expression point for the gene investigated) and standard errors. Primers used for RT-qPCR are listed in Table S1 and amplification efficiency based on serial dilution was greater than $90 \%$ for each primer pair (Table S1). Table S2 shows the expression data of UBC21 (At5G25760) used as the reference gene at 0, 48 and $96 \mathrm{~h}$ protoplast culture.

\subsection{Immunoblotting}

For immunoblotting experiments, Col-0, act2-1, and act7-1 protoplasts were cultured for the indicated times and $1 \times 10^{6}$ protoplasts pelleted and dissolved in SDS sample buffer and heated to $95{ }^{\circ} \mathrm{C}$ for $5 \mathrm{~min}$. After centrifugation at $10,000 \times g$ for $5 \mathrm{~min}$, equal volumes of supernatant from each extract were loaded into wells of $10 \%$ polyacrylamide gels. After electrophoresis and transfer to nitrocellulose, the blots were blocked with $5 \%(w / v)$ skim milk for $1 \mathrm{~h}$, washed in Tris-buffered saline solution then probed with C4 anti-chicken gizzard actin (MP Biomedicals, Irvine, CA, USA; [27]) for $2 \mathrm{~h}$. After washing with Tris-buffered saline (TBS), the nitrocellulose was incubated with goat anti-mouse secondary antibody coupled to alkaline phosphatase. Color development was by Western 
Blue (Promega, Madison, WI, USA) and individual band intensities were quantified from scanned images using ImageJ. Normalized band intensities were determined by comparison to total tubulin levels (mouse monoclonal anti- $\alpha$-tubulin, clone B512, Sigma) assessed on duplicate nitrocellulose blots and processed in parallel.

Supplementary Materials: The following are available online at http://www.mdpi.com/2223-7747/9/2/225/s1, Table S1: List of primers used in this study. Table S2: Expression of $U B C 21$ in isolated protoplasts cultured for 0, 48 and $96 \mathrm{~h}$.

Author Contributions: M.B.S., R.J.R., and D.W.M. designed the experiments; M.B.S. and D.A.C. performed experimental analyses; M.B.S., D.A.C., and D.W.M. drafted the manuscript; D.A.C., R.J.R., and D.W.M. approved the final manuscript; M.B.S. passed away before completion of this manuscript. All authors have read and agreed to the published version of the manuscript.

Funding: Supported in part from the Australian Research Council Centre of Excellence grant to R.J.R (project number CEO348212) and the Faculty of Science, University of Newcastle funding to D.W.M.

Acknowledgments: The authors thank Richard Meagher (University of Georgia, USA) for generously supplying the $A C T$ transgenic lines used in this study, and Masamitsu Wada (Kyushu University, Japan) for the chup1 mutant.

Conflicts of Interest: The authors declare no conflict of interest.

\section{References}

1. Ng, R.; Abelson, J. Isolation and sequence of the gene for actin in Saccharomyces cerevisiae. Proc. Natl. Acad. Sci. USA 1980, 77, 3912-3926. [CrossRef] [PubMed]

2. McDowell, J.M.; Huang, S.; McKinney, E.C.; An, Y.-Q.; Meagher, R.B. Structure and evolution of the actin gene family in Arabidopsis thaliana. Genetics 1996, 142, 587-602. [PubMed]

3. Meagher, R.B.; McKinney, E.C.; Kandasamy, M.K. Isovariant dynamics expand and buffer the responses of complex systems: The diverse plant actin gene family. Plant Cell 1999, 11, 995-1005. [CrossRef] [PubMed]

4. Kandasamy, M.K.; McKinney, E.C.; Roy, E.; Meagher, R.B. Plant vegetative and animal cytoplasmic actins share functional competence for spatial development with protists. Plant Cell 2012, 24, 2041-2057. [CrossRef]

5. S̆lajcherová, K.; Fišerová, J.; Fischer, L.; Schwarzerová, K. Multiple actin isotypes in plants: Diverse genes for diverse roles? Front. Plant Sci. 2012, 3, 226. [CrossRef] [PubMed]

6. Perrin, P.J.; Ervasti, J.M. The actin gene family: Function follows isoform. Cytoskeleton 2010, 67, 630-634. [CrossRef] [PubMed]

7. Röper, K.; Mao, Y.; Brown, N.H. Contribution of sequence variation in Drosophila actins to their incorporation into actin-based structures in vivo. J. Cell Sci. 2005, 118, 3937-3948. [CrossRef] [PubMed]

8. Brault, V.; Reedy, M.C.; Sauder, U.; Kammerer, R.A.; Aebi, U.; Schoenenberger, C.-A. Substitution of flight muscle-specific actin by human $\beta$-cytoplasmic actin in the indirect flight muscle of Drosophila. J. Cell Sci. 1999, 112, 3627-3639.

9. Kandasamy, M.K.; Gilliland, L.U.; McKinney, E.C.; Meagher, R.B. One plant actin isovariant, ACT7, is induced by auxin and required for normal callus formation. Plant Cell 2001, 13, 1541-1554. [CrossRef] [PubMed]

10. Gilliland, L.U.; Pawloski, L.C.; Kandasamy, M.K.; Meagher, R.B. Arabidopsis actin gene ACT7 plays an essential role in germination and root growth. Plant J. 2003, 33, 319-328. [CrossRef] [PubMed]

11. Nakamura, M.; Claes, A.R.; Grebe, T.; Hermkes, R.; Viotti, C.; Ikeda, Y.; Grebe, M. Auxin and ROP GTPase signalling of polar nuclear migration in root epidermal hair cells. Plant Physiol. 2018, 176, 378-391. [CrossRef]

12. Ringli, C.; Baumberger, N.; Diet, A.; Frey, B.; Keller, B. ACTIN2 is essential for bulge site selection and tip growth during root hair development of Arabidopsis. Plant Physiol. 2002, 129, 1464-1472. [CrossRef] [PubMed]

13. Kandasamy, M.K.; McKinney, E.C.; Meagher, R.B. A single vegetative actin isovariant overexpressed under the control of multiple regulatory sequences is sufficient for normal Arabidopsis development. Plant Cell 2009, 21, 701-718. [CrossRef] [PubMed]

14. Kijima, S.T.; Hirose, K.; Kong, S.G.; Wada, M.; Uyeda, T.Q.P. Distinct biochemical properties of Arabidopsis thaliana actin isoforms. Plant Cell Physiol. 2016, 57, 46-56. [CrossRef] [PubMed]

15. Kijima, S.T.; Staiger, C.J.; Katoh, K.; Nagasaki, A.; Ito, K.; Uyeda, T.Q.P. Arabidopsis vegetative actin isoforms, AtACT2 and AtACT7, generate distinct filament arrays in living plant cells. Sci. Rep. 2018, 8, 4381. [CrossRef] 
16. Thitamadee, S.; Tuchihara, K.; Hashimoto, T. Microtubule basis for left-handed helical growth in Arabidopsis. Nature 2002, 417, 193-196. [CrossRef]

17. Ishida, T.; Kaneko, Y.; Iwano, M.; Hashimoto, T. Helical microtubule arrays in a collection of twisting tubulin mutants of Arabidopsis thaliana. Proc. Natl. Acad. Sci. USA 2007, 104, 8544-8549. [CrossRef]

18. Sunohara, H.; Kawai, T.; Shimizu-Sato, S.; Sato, Y.; Sato, K.; Kitano, H. A dominant mutation of TWISTED DWARF1 encoding an $\alpha$-tubulin protein causes severe dwarfism and right helical growth in rice. Genes Gene Syst. 2009, 84, 209-218. [CrossRef] [PubMed]

19. Jöst, M.; Esfeld, K.; Burian, A.; Cannarozzi, G.; Chanyalew, S.; Kuhlemeier, C.; Assefa, K.; Tadele, Z. Semi-dwarfism and lodging tolerance in tef (Eragrostis tef) is linked to a mutation in the $\alpha$-Tubulin 1 gene. J. Exp. Bot. 2015, 66, 933-944. [CrossRef]

20. Vaškebová, L.; Šamaj, J.; Ovečka, M. Single-point ACT2 gene mutation in the Arabidopsis root hair mutant der1-3 affects overall actin organization, root growth and plant development. Ann. Bot. 2018, 122, 889-901. [CrossRef]

21. Kato, T.; Morita, M.T.; Tasaka, M. Defects in dynamics and functions of actin filament in Arabidopsis caused by the dominant-negative actin fiz1-induced fragmentation of actin filament. Plant Cell Physiol. 2010, 51, 333-338. [CrossRef]

22. Sheahan, M.B.; Rose, R.J.; McCurdy, D.W. Organelle inheritance in plant cell division: The actin cytoskeleton is required for unbiased inheritance of chloroplasts, mitochondria and endoplasmic reticulum in dividing protoplasts. Plant J. 2004, 37, 379-390. [CrossRef] [PubMed]

23. Sheahan, M.B.; McCurdy, D.W.; Rose, R.J. Mechanisms of organelle inheritance in dividing plant cells. In Molecular Cell Biology of the Growth and Differentiation of Plant Cells; Rose, R.J., Ed.; CRC Press: Boca Raton, FL, USA, 2016; pp. 66-88.

24. Kandasamy, M.K.; Meagher, R.B. Actin-organelle interaction: Association with chloroplast in Arabidopsis leaf mesophyll cells. Cell Motil. Cytoskel. 1999, 44, 110-118. [CrossRef]

25. Sheahan, M.B.; Staiger, C.J.; Rose, R.J.; McCurdy, D.W. A green fluorescent protein fusion to actin-binding domain 2 of Arabidopsis fimbrin highlights new features of a dynamic actin cytoskeleton in live plant cells. Plant Physiol. 2004, 136, 3968-3978. [CrossRef] [PubMed]

26. Oikawa, K.; Kasahara, M.; Kiyosue, T.; Kagawa, T.; Suetsugu, N.; Takahashi, F.; Kanegae, T.; Niwa, Y.; Kadota, A.; Wada, M. CHLOROPLAST UNUSUAL POSITIONING1 is essential for proper chloroplast positioning. Plant Cell 2003, 15, 2805-2815. [CrossRef]

27. Gilliland, L.U.; Kandasamy, M.K.; Pawloski, L.C.; Meagher, R.B. Both vegetative and reproductive actin isovarients complement the stunted root hair phenotype of the Arabidopsis act2-1 mutation. Plant Physiol. 2002, 130, 2199-2209. [CrossRef] [PubMed]

28. Lessard, J.L. Two monoclonal antibodies to actin: One muscle selective and one generally reactive. Cell Motil. Cytoskel. 1989, 10, 349-362. [CrossRef] [PubMed]

29. Kadota, A.; Yamada, N.; Suetsugu, N.; Hirose, M.; Saito, C.; Shoda, K.; Ichikawa, S.; Kagawa, T.; Nakano, A.; Wada, M. Short actin-based mechanism for light-directed chloroplast movement in Arabidopsis. Proc. Natl. Acad. Sci. USA 2009, 106, 13106-13111. [CrossRef] [PubMed]

30. Kong, S.-G.; Wada, M. New insights into dynamic actin-based chloroplast photorelocation movement. Mol. Plant 2011, 4, 771-781. [CrossRef] [PubMed]

31. Collings, D.A.; Lill, A.W.; Himmelspach, R.; Wasteneys, G.O. Hypersensitivity to cytoskeletal antagonists demonstrates microtubule-microfilament cross-talk in the control of root elongation in Arabidopsis thaliana. New Phytol. 2006, 170, 275-290. [CrossRef]

32. Muller, P.Y.; Janovjak, H.; Miserez, A.R.; Dobbie, Z. Processing of gene expression data generated by quantitative real-time RT-PCR. Biotechniques 2002, 32, 1372-1379. [PubMed]

33. Simon, P. Q-Gene: Processing quantitative real-time RT-PCR data. Bioinformatics 2003, 19, 1439-1440. [CrossRef] [PubMed]

34. Q-Gene software. Available online: http://download.gene-quantification.info/ (accessed on 5 January 2020).

(C) 2020 by the authors. Licensee MDPI, Basel, Switzerland. This article is an open access article distributed under the terms and conditions of the Creative Commons Attribution (CC BY) license (http://creativecommons.org/licenses/by/4.0/). 